\title{
Stability Analysis of Multi-Inverters Grid-Connected System Using Improved Current Source Impedance Ratio Criterion
}

\author{
Hu Guozhen ${ }^{1,2}$, Mao Ling ${ }^{1}$, Liu Jun ${ }^{1}$, Zhang Lei ${ }^{1}$, Fang Zhi Jian ${ }^{2}$ \\ ${ }^{I}$ School of Electrical and Electronic Information Engineering, Hubei Polytechnic University, \\ Huangshi, China, 435003 \\ ${ }^{2}$ School of Electrical Engineering, Wuhan University, \\ Wuhan, China, 430072 \\ huguozhen@hbpu.edu.cn
}

\begin{abstract}
Stability analysis of multi-inverters grid-connected system in electric vehicles power station is presented in this paper. An equivalent circuit model of the multi-inverters grid-connected system is built based on electrical network theory. Then an improved current source impedance criterion is proposed to analyse the stability of the system which is mainly related to the number of grid connected inverters, grid impedance and output impedance of the inverters. Finally, simulation results are given to verify the effectiveness of the multi-inverters model and the improved current source impedance criterion.
\end{abstract}

Index Terms-Multi-inverters; Grid-connected; Impedance criterion; Stability analysis.

\section{INTRODUCTION}

Recently, Electric vehicles (EVs) are becoming an ecologically attractive alternative to gasoline driven cars. Large-scale integration of the EVs can serve as a distributed and mobile energy source in the electricity market, which construct a new form of energy feedback system [1], [2]. EVs power station is an intelligent power station with good application prospects. When the energy generated by power systems is insufficient, the station can deliver energy from the local EVs storage to grid [3], [4].

Conventional energy feedback system connected to the grid with a single inverter in Point of Common Coupling (PCC), which required storage battery centrally stored and caused a higher grid-connected inverter power capacity. In the EVs power station, multi-points connection to grid is more convenient for the large number of inverters. And the local use of the EVs storage battery can also reduce the power capacity of the inverter.

Usually, the effect of mutual coupling is small in a strong grid even with a large number of inverters at multi-points connection to grid. However, in the EVs power station, the energy feedback system is connected to the grid through medium or low voltage transformer causing a weak grid

Manuscript received 27 May, 2018; accepted 16 December, 2018.

This research was funded by a grant from China Postdoctoral Science Foundation (168154) and Natural Science Foundation of Hubei Province (2017CFB195, D20164501) which would bring stability problem due to the leakage and line inductors. In order to solve this problem, some papers have been published. In [5] a simplified equivalent method has been proposed based on the small signal model of multi-inverters parallel system. In [6] equivalent impedance model of the multi-inverters parallel system has been established, and the authors concluded that the resonance peaks of multi-inverters parallel system changed with the number of parallel inverters. The problem of power quality in distributed generation system has been analysed in [7] caused by series-parallel resonance of a large number of inverters. Furthermore, the literature [8] pointed that multi-inverters grid-connected system would generate grid voltage harmonic distortion and grid-connected current oscillation in multi-inverters grid-connected distributed generation system.

In this paper, a simplified equivalent model of the multi-inverters grid-connected system is established based on the circuit network theory. The control performance and stability of the system are analysed by improving traditional voltage source impedance criterion to current source impedance ratio criterion. Finally, the simulation results are given to verify the effectiveness of multi-inverter model and the improved current source impedance criterion.

\section{II.MODEL OF MULTI-INVERTERS GRID-CONNECTED SYSTEM}

In the EVs power station, the input of the energy feedback system is storage battery and the output is connected to the three-phase grid with output filters. In order to simplify the analysis, the model of multi-inverters grid-connected system can be equivalent as Fig. 1 [9].

In Fig. 1, LCL filters are used in the system due to their effective switching harmonic attenuation. $Z_{11}, Z_{21}, \ldots . Z_{n 1}$ are inverter side inductor impedances; $Z_{12}, Z_{22}, \ldots . Z_{n 2}$ are grid side inductor impedances; $Z_{13}, Z_{23}, \ldots . Z_{n 3}$ are capacitor impedances; $Z_{g}$ is the grid impedance; $V_{g}$ is the grid voltage, $i_{01} \ldots i_{0 n}$ is the inverter side inductor current. Matrix form of the transfer function between the grid side inductor current $i_{11} \ldots i_{1 n}$ and the output voltage of inverter $v_{01} \ldots v_{0 n}$ can be presented as follows: 


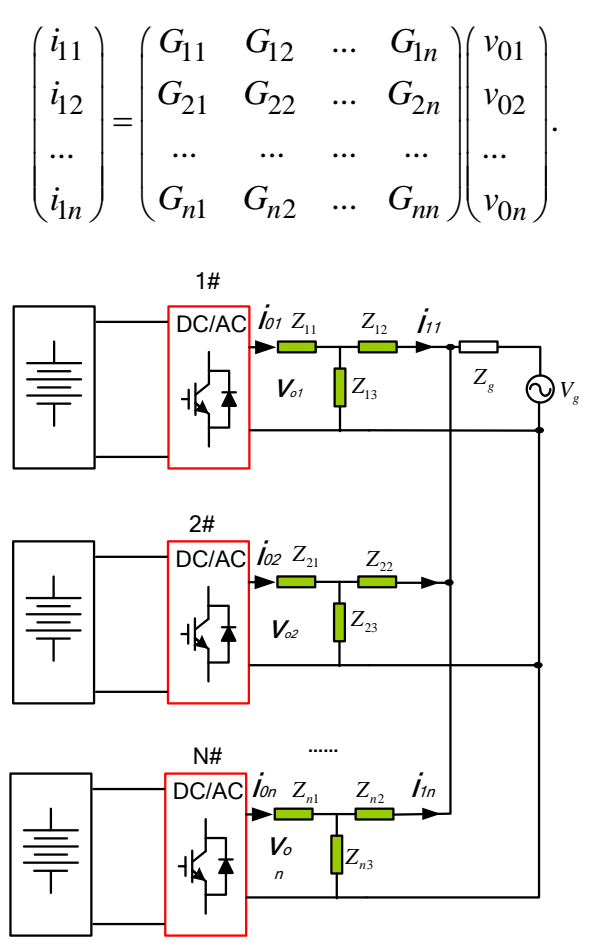

Fig. 1. Equivalent model of multi-inverters grid-connected system.

$G(s)$ is the coupling function among multi-inverters grid-connected system in (1). Due to the coupling of parameters in multi-inverters system, it is difficult to accurately obtain the expression of $G(s)$. Considering the maturity of production and manufacturing process, assume the output characteristics of inverters are consistent. Therefore all diagonal elements of $G(s)$ are equal $\left\{\begin{array}{l}G_{11}=G_{22}=\ldots=G_{n n} \\ G_{12}=G_{21}=\ldots=G_{n 2}=G_{n 1} . G(s) \text { can be expressed }\end{array}\right.$ as a symmetric matrix, and (1) can be rewritten as follows:

$$
\left(\begin{array}{l}
i_{11} \\
i_{12} \\
\ldots \\
i_{1 n}
\end{array}\right)=\left(\begin{array}{cccc}
G_{11} & G_{12} & \ldots & G_{12} \\
G_{12} & G_{11} & \ldots & G_{12} \\
\ldots & \ldots & \ldots & \ldots \\
G_{12} & G_{12} & \ldots & G_{11}
\end{array}\right)\left(\begin{array}{l}
v_{01} \\
v_{02} \\
\ldots \\
v_{0 n}
\end{array}\right) .
$$

According to (2), the relationship between the $\mathrm{n}$-th grid side inductor current $i_{1 n}$ and the n-th output voltage of inverter $v_{0 n}$ is given in

$$
i_{1 n}=G_{11} v_{0 n}+G_{12}\left(v_{01}+\ldots+v_{0 n-1}\right) .
$$

Considering the output characteristics of multi-inverters are voltage source, the influence of nonlinear modulation can be ignored. The transfer function $G_{m}(s)$ between $i_{1 n}$ and $v_{0 n}$ can be presented as follows

$$
G_{m}(s)=\frac{i_{1 n}}{v_{0 n}} \approx G_{11}+(n-1) G_{12},
$$

where $n \neq 1$. In (4), $G_{11}$ reflects the relationship between the output voltage and the output current of single inverter. $G_{12}$ reflects the relationship between the output voltage of other inverters and the output current of the single inverter. According to superposition theorem of the circuit network, $G_{11}, G_{12}$ and $G_{m}(s)$ can be obtained respectively.

1. Assuming the output voltage of the inverters is zero, and $\left\{\begin{array}{l}Z_{11}=Z_{21}=\ldots=Z_{n 1}=Z_{1} \\ Z_{12}=Z_{22}=\ldots=Z_{n 2}=Z_{2}, \text { Fig. } 1 \text { can be equivalent to } \\ Z_{13}=Z_{23}=\ldots=Z_{n 3}=Z_{3}\end{array}\right.$

Fig. 2, and $G_{11}$ can be obtained

$$
\begin{gathered}
G_{11}=\frac{i_{1 n}^{\prime}}{v_{0 n}^{\prime}}= \\
=\frac{Z_{1}\left(Z_{3}+Z_{2}\right)\left(Z_{3}+Z_{2}+n Z_{g}\right)+Z_{3}\left(Z_{2}^{2}+(n-1) Z_{3} Z_{g}+Z_{2}\left(Z_{3}+n Z_{g}\right)\right)}{\left(Z_{2} Z_{3}+Z_{1}\left(Z_{2}+Z_{3}\right)\right)\left(Z_{3}\left(Z_{2}+n Z_{g}\right)+Z_{1}\left(Z_{3}+Z_{2}+n Z_{g}\right)\right)},
\end{gathered}
$$

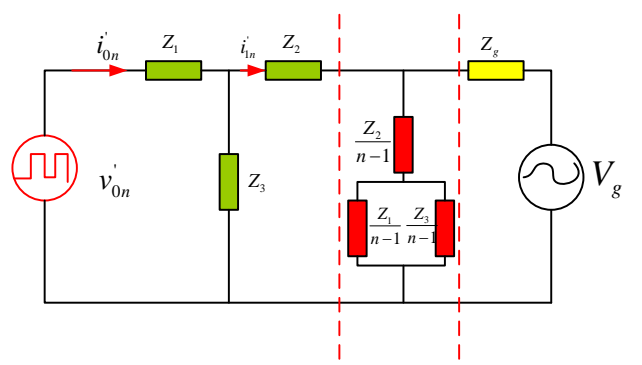

Fig. 2. Equivalent model for calculating $G_{11}$

2. Assuming the output voltage of inverters is zero, Fig. 1 can be equivalent to the Fig. 3 , and $G_{12}$ can be obtained

$$
\begin{gathered}
G_{12}=\frac{i_{1 n}^{\prime}}{v_{0 n}^{\prime \prime}}= \\
=\frac{Z_{3}^{2} Z_{g}}{\left(Z_{2} Z_{3}+Z_{1}\left(Z_{2}+Z_{3}\right)\right)\left(Z_{3}\left(Z_{2}+n Z_{g}\right)+Z_{1}\left(Z_{3}+Z_{2}+n Z_{g}\right)\right)} .
\end{gathered}
$$

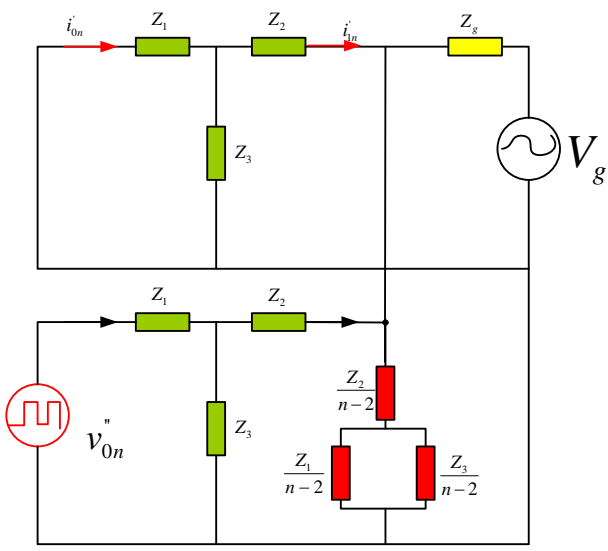

Fig. 3. Equivalent model for calculating $G_{12}$.

3. Based on (5) and (6), $G_{m}$ can be obtained

$G_{m}(s)=\frac{i_{0 n}}{v_{0 n}} \approx \frac{Z_{3}+Z_{2}+n Z_{g}}{Z_{3}\left(Z_{2}+n Z_{g}\right)+Z_{1}\left(Z_{3}+Z_{2}+n Z_{g}\right)}$.

As shown in (7), the difference between equivalent model of the multiple grid-connected inverters and the single grid-connected inverter is $n \cdot Z_{g}$. So the multi-inverters can be seen as a single inverter with $\mathrm{n}$ time's grid impedance. 


\section{IMPROVED CURRENT SOURCE IMPEDANCE RATIO CRITERION FOR STABILITY ANALYSIS}

Traditional stability analysis usually uses loop gain analysis method based on classical control theory. And closed-loop transfer function of converters is established according to small signal model. When the number of the converters is large, the interaction among individually designed power modules may cause the instability of the whole system. And the orders of the closed-loop transfer function could increase exponentially with the numbers of converters rising, which causes loop gain analysis method become more complex.

As a result, defining impedance specification is an effective way to analyse stability for this system. This method realizes the decoupling of the power supply and the load. Only the input and output impedance characteristics of source and load need to be considered. Traditional voltage source impedance ratio criterion is mainly based on (8). $Z_{s}$ is the impedance of power supply, $Z_{i}$ is the load impedance, $V_{s}$ is the voltage of power supply, $V_{i}$ is the voltage of the load. The impedance ratio $T_{m}=Z_{s} / Z_{i}$ is regarded as loop gain to analyse system stability

$$
V_{i}=V_{s} \times \frac{Z_{i}}{Z_{s}+Z_{i}}=V_{s} \times \frac{1}{1+\frac{Z_{s}}{Z_{i}}} .
$$

However, the traditional impedance ratio criterion is mainly suitable for the applications that characteristics of the power supply and load are voltage source [10], [11]. The grid-connected system only can be regarded as current source in the view of the grid, and the grid-connected current must maintain the same frequency and phase with the grid to ensure the stability of the system. The traditional impedance criterion is no longer applicable. In this paper an improved current source impedance ratio criterion has been proposed.

Based on the equivalent transformation of circuit networks as shown in Fig. 4, the source is grid-connected inverters and the load is the grid. Therefore, improved current source impedance criterion can be obtained as (9). $I_{s}$ is the equivalent current source. $I_{L}$ is the equivalent load current

$$
I_{L}=I_{s} \times \frac{Z_{s}}{Z_{s}+Z_{g}}=I_{s} \times \frac{1}{1+\frac{Z_{g}}{Z_{s}}} .
$$

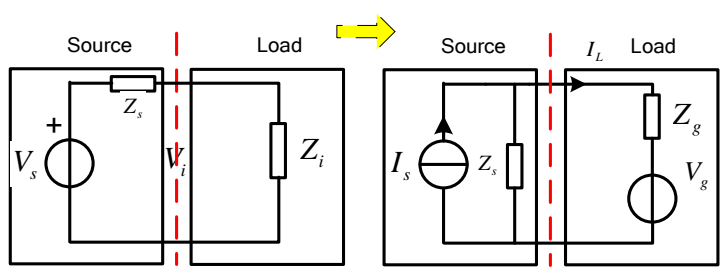

Fig. 4. Equivalent model of improved current source impedance criterion.

Compared with (8) and (9), the impedance ratio based on current source mode is $T_{m}=Z_{g} / Z_{s}$. Considering the disturbance of grid, the output current can be written as follows

$I_{L}=I_{s} \times \frac{Z_{s}}{Z_{s}+Z_{g}}-\frac{V_{g}}{Z_{s}+Z_{g}}=\frac{1}{1+\frac{Z_{g}}{Z_{s}}}\left[I_{s}-\frac{1}{Z_{s}} V_{g}\right]$.

Equation (10) is the same with the small signal model of grid-connected inverter. Therefore, the impedance ratio $T_{m}=Z_{g} / Z_{s}$ can be regarded as stability criterion of the grid-connected system.

The stability can be analysed by Nyquist criterion. In order to overcome the conservatism and forbidden region of the improved current source criterion, the following two points are mainly considered.

1. When $\left|T_{m}\right| \leq 1$, the system can keep stability in full frequency range.

2. When $\left|T_{m}\right|>1$, the stability analysis should combine with the phase margin. Due to the existence of grid impedance and other reactive elements, resonance and harmonic distortion is easy to appear in AC system, which is different from DC system.

\section{Stability Analysis of Multi-InVERTERS GRID-CONNECTED SYSTEM}

\section{A. Impedance Characteristics of LCL Grid-connected System \\ 1) Equivalent Grid Impedance}

Due to the nonlinear elements of power loads, it is difficult to obtain accurate model of the grid. Usually it is approximately equivalent to resistance $R_{g}$ and inductance $L_{g}$ in series. The resistance-inductance equivalent model consists with actual measurement of the grid in high frequency range. And the characteristic in low frequency range is mainly decided by transformer leakage reactance and line impedance of middle and low voltage network to PCC. Therefore, the value of grid impedance $Z_{g}$ can be estimated according to the impedance voltage ratio of the transformer. Equivalent model of the grid is given as

$$
Z_{g}=R_{g}+s L_{g}
$$

\section{2) Output Impedance of LCL Grid-Connected Inverters}

In order to eliminate the influence of resonance caused by LCL grid-connected filters, passive damping and active damping are widely used in practice. Active damping with virtual resistance algorithm can effectively suppress resonant peak caused by LCL filters and improve system efficiency [12], which is used to analyse the output impedance of the grid-connected inverters in this paper.

The dual-loop control diagram of grid-connected inverter with virtual impedance is shown in Fig. 5.

The outer loop is grid-connected current control and the inner loop is capacitor current control. And the open-loop transfer function of the system is given as

$$
G_{\text {open }}=\frac{K_{p w m}\left(K_{p} s+K_{i}\right)}{s^{2}\left(s^{2} L_{1} L_{2} C+s K_{c} L_{2} C+L_{1}+L_{2}\right)},
$$


where $L_{1}$ is the inverter side inductor, $L_{2}$ is the grid side inductor, $C$ is the parallel capacitor, $K_{p w m}$ is the system gain, $K_{p}$ and $K_{i}$ are parameters of PI controller $G_{0}(s) . K_{c}$ is the virtual resistance feedback gain. $i_{1}$ is the grid-connected current, $i_{r}$ is given current of grid-connected current, $i_{c}$ is capacitor current, $v_{c}$ is capacitor voltage.

$$
\left\{\begin{array}{l}
{\left[-i_{1} G_{0}(s) K_{p w m}-K_{c} K_{p w m} i_{c}-v_{c}\right] \times \frac{1}{Z_{1}}-i_{L 2}=i_{c},} \\
{\left[v_{c}-v_{o}\right] \frac{1}{Z_{2}}=i_{L 2},} \\
i_{c} \times Z_{3}=v_{c} .
\end{array}\right.
$$

According to Fig. 5, (13) can be obtained as follows:

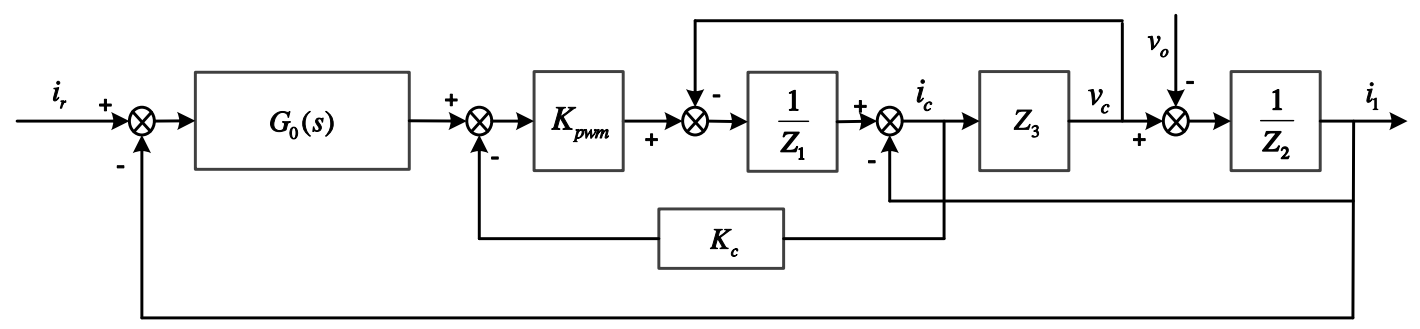

Fig. 5. Dual-loop control diagram of grid-connected inverter.

According to (12) and (13), the output impedance $Z_{o}$ can be obtained

$$
\begin{gathered}
Z_{o}=\frac{v_{o}}{-i_{1}}=Z_{2}+\frac{\left(G_{0}(s) K_{p w m}+Z_{1}\right) Z_{3}}{Z_{1}+Z_{3}+K_{c} K_{p w m}}= \\
=\frac{\left(Z_{1}+K_{c} K_{p w m}\right) Z_{2}+\left(G(s) K_{p w m}+Z_{1}+Z_{2}\right) Z_{3}}{Z_{3}+Z_{1}+K_{c} K_{p w m}} .
\end{gathered}
$$

When internal resistance of capacitor and inductor are ignored, (14) can be equivalent to (15)

$$
Z_{o}=\frac{L_{1} L_{2} C s^{4}+s^{3} C L_{2} K_{c} K_{p w m}+s^{2}\left(L_{1}+L_{2}\right)+K_{p} K_{p w m} s+K_{i} K_{p w m}}{L_{1} C s^{3}+K_{c} K_{p w m} C s^{2}+s}
$$

\section{B. Stability Analysis}

According to parameters in Table I, frequency characteristics of $Z_{o}$ and $Z_{g}$ are shown in Fig. 6 in three cases $(\mathrm{n}=1,20,50)$.

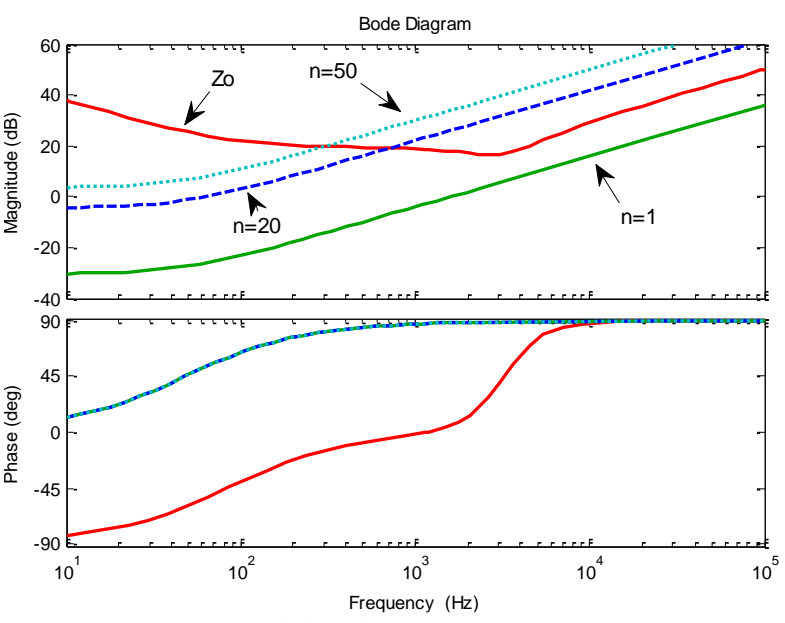

Fig. 6. Frequency characteristics of $Z o$ and $Z g$.

The performance of the multi-inverters grid-connected system is analysed based on the current source impedance criterion. $Z_{o}$ can be regard as the impedance of power supply in Section III. The impedance ratio $T_{m}$ is given by

$$
\begin{gathered}
T_{m}=\frac{n^{*} Z_{g}}{Z_{o}}= \\
=\frac{n L_{g}}{L_{2}} \times \frac{s\left(s+R_{g} / L_{g}\right)\left(s^{2}+s K_{c} K_{p w m} / L_{1}+1 / L_{1} C\right)}{s^{4}+s^{3}\left(K_{c} K_{p w m} / L_{1}\right)+s^{2}\left(L_{1}+L_{2}\right) / L_{1} L_{2} C+s\left(K_{p} K_{p w m} / L_{1} L_{2} C\right)+K_{i} K_{p w m} / L_{1} L_{2} C} .
\end{gathered}
$$

TABLE I. SIMULATION PARAMETERS

\begin{tabular}{|c|c|}
\hline Parameters & Value \\
\hline DC voltage & $390 \mathrm{~V}$ \\
\hline$L_{l}=L_{2}$ & $0.5 \mathrm{mH}$ \\
\hline$C$ & $5 \mathrm{uF}$ \\
\hline$K_{p}$ & 9 \\
\hline$K_{i}$ & 12500 \\
\hline$L_{g}$ & $0.3 \mathrm{mH}$ \\
\hline
\end{tabular}

The amplitude-frequency characteristics of $T_{m}$ in three cases $(\mathrm{n}=1,20,50)$ are shown in Fig. 7. According to impedance characteristics shown in (16), the characteristic in high frequency range is mainly decided by $n L_{g} / L_{2}$, while the coupling point between multi-inverters output impedance and grid impedance often appears in middle and low frequency range. Therefore, stable operating characteristics of the system should consider the phase margin.

It can be seen from Fig. 7 that the stability and harmonic quality meets requirement when $\mathrm{n}=1$ and $\left|T_{m}\right|$ is less than 1 in the range of full frequency. When $n=20$, impedance crossover frequency is about $800 \mathrm{~Hz}$ and the phase margin is about $90^{\circ}$, also the system has good stability. When $\mathrm{n}=50$, impedance crossover frequency is about $300 \mathrm{~Hz}$, the phase margin is about $80^{\circ}$. With the increasing of the number of grid-connected inverters, the impedance crossover frequency and phase margin will be decreased regularly. Furthermore, the low-frequency oscillation would be leads to excessive harmonics distortion and resonance. 


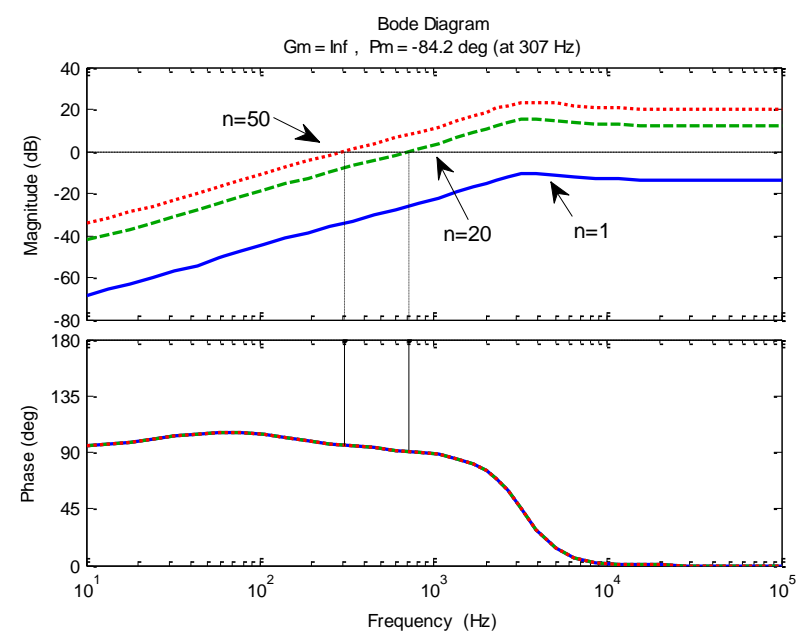

Fig. 7. Frequency characteristics of impedance ratio $T_{m}$.

Nyquist curves in three cases $(n=1,20,50)$ are shown in Fig. 8. The phase margin of multi-inverters grid-connected system is decreased with the increasing of the number of grid-connected inverters. When it is decreased to a certain degree, the system will be unstable due to resonance.

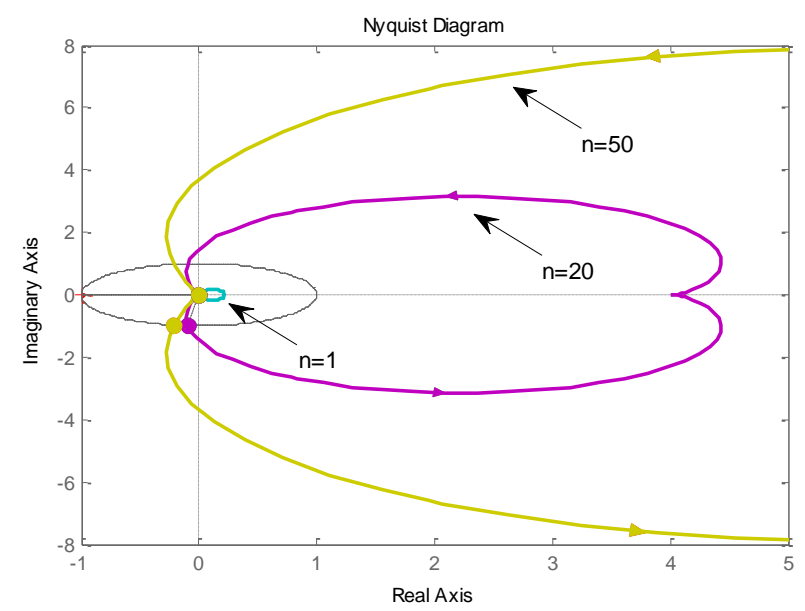

Fig. 8. Nyquist curves of impedance ratio $T_{m}$.

\section{V.SIMULATION RESULTS}

Simulation results are present to verify the theoretical analysis. Simulation parameters are shown in Table I.

In order to verify the accuracy of multi-inverters grid-connected equivalent model, two different simulating ways are implemented. And grid-connected current steps at $0.045 \mathrm{~s}$ to analyse dynamic characteristics of the model. $I_{\text {oeq }}$, $V_{\text {seq }}$ are the output current and voltage of the equivalent model proposed in Section II. $I_{o}, V_{\mathrm{s}}$ are the output current and voltage of multi--paralleled inverters.

When the impedance $Z_{g}^{\prime}$ of multi-inverters grid-connected system is equal to $n$ time's grid impedance, it can be seen from Fig. 9 that the equivalence keeps correspondence with these two cases.

As shown in Fig. 10, when the grid impedance is not equal to n time's grid impedance, the system does not meet the proposed equivalent relationship and output characteristics are quite different in these two cases.

Also simulation results are present to verify the improved current source impedance ratio criterion. Simulation waveform of a single LCL grid-connected inverter $(n=1)$ with virtual resistance algorithm is shown in Fig. 11. The power quality of output voltage and grid-connected current is higher.
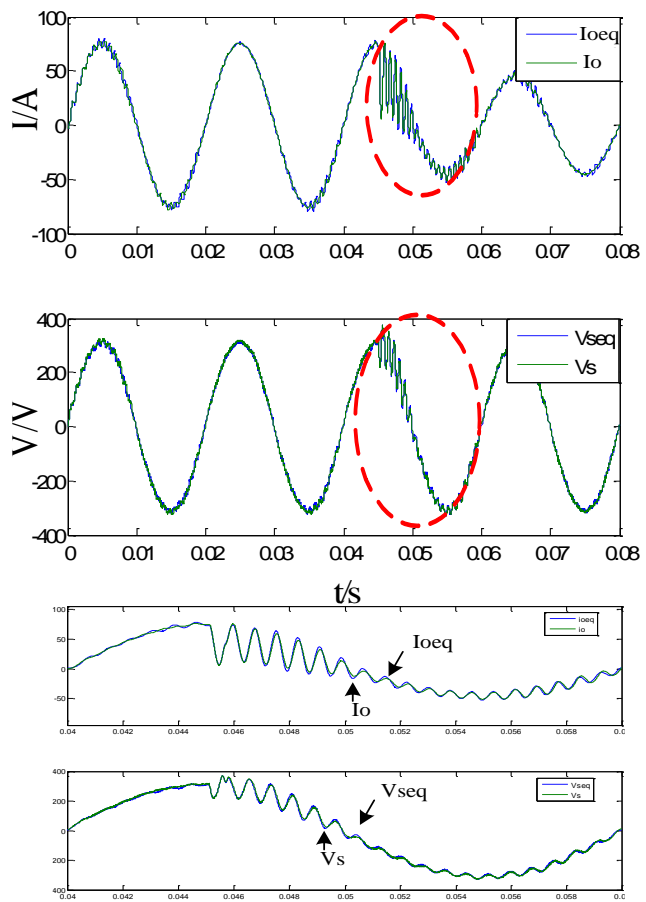

Fig. 9. Simulation waveforms $\left(Z_{g}^{\prime}=n Z_{g}\right)$.
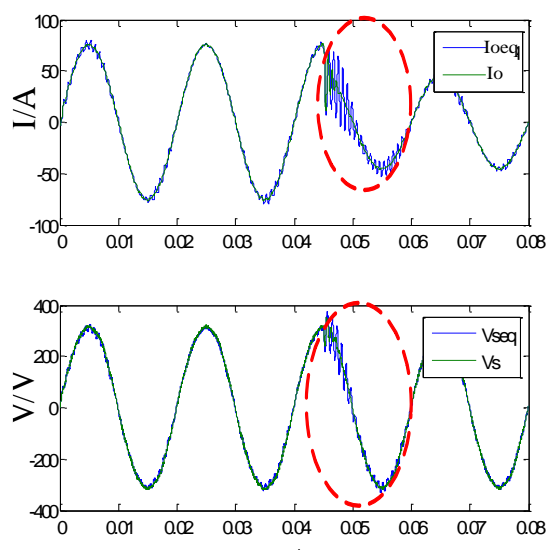

ts

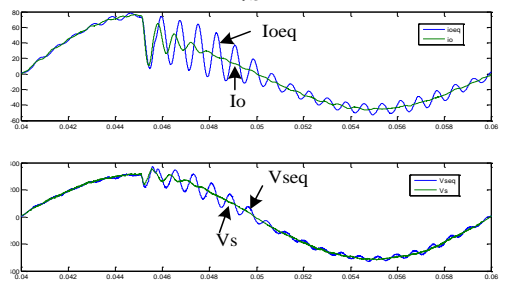

Fig. 10. Simulation waveforms $\left(Z_{g}^{\prime} \neq n Z_{g}\right)$.

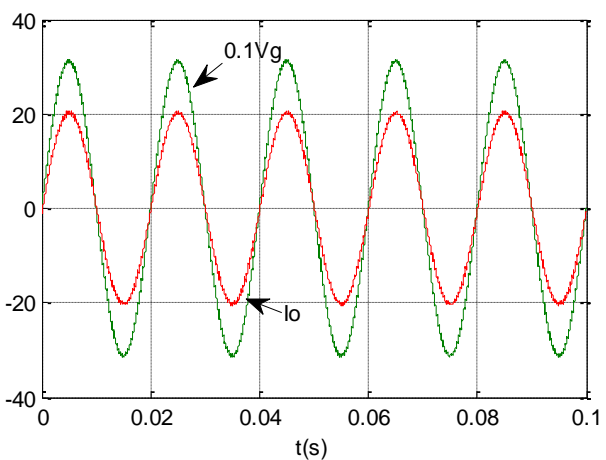

Fig. 11. Simulation waveforms of LCL grid-connected system $(n=1)$. 
Simulation waveforms of grid-connected inverters $(\mathrm{n}=20)$ are shown in Fig. 12. The system stability is good and the Total Harmonic Distortion (THD) of grid-connected current is $0.69 \%$.
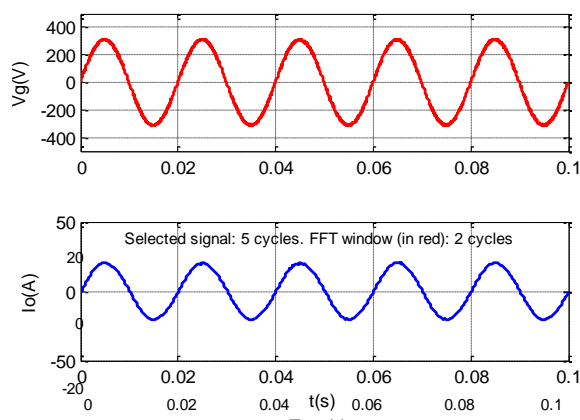

(致) (s)

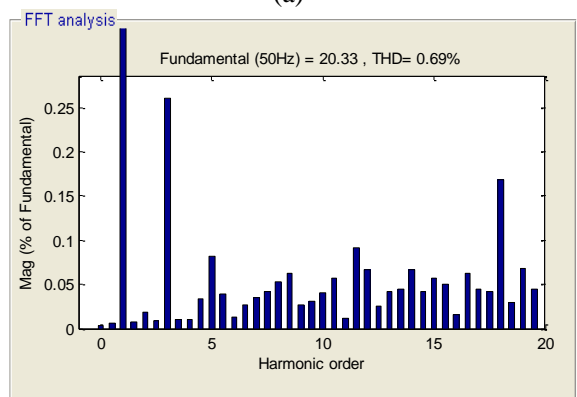

(b)

Fig. 12. Simulation waveforms of LCL grid-connected system $(n=20)$ : (a) Grid-connected voltage and current waveforms; (b) THD of grid-connected current.

Simulation waveforms of grid-connected inverters $(n=50)$ are shown in Fig. 13. The system is basically stable, but the THD of grid-connected current is $6.31 \%$. THD exceeds grid connection requirements seriously, especially the low order harmonic current. This situation mainly caused by the lower impedance crossover frequency. It is difficult to suppress by adjusting control bandwidth or algorithm.
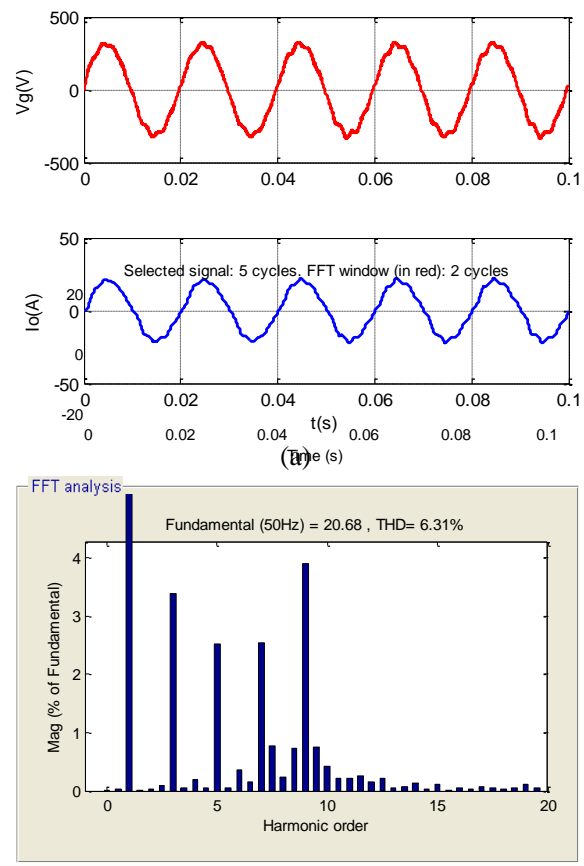

(b)

Fig. 13. Simulation waveforms of LCL grid-connected system $(n=50)$ : a) Grid-connected voltage and current waveforms; b) THD of grid-connected current.
It can be seen that simulation results are consistent with the analysis in Section IV. When the number of paralleled-inverters is excessive, the power quality of grid-connected current would be poor. Therefore, the number of the inverters in EVs power station needs to be limited when multi-inverters are connected to the grid at the same time.

\section{CONCLUSIONS}

In this paper, multi-inverters grid-connected system for EVs Power Station is studied. The equivalent model of the multi-inverters grid-connected system is obtained through the equivalent analysis with circuit network theory. Improved current source impedance is proposed to analysis stability of multi-inverters grid-connected system. And the resonance problem of multi-inverters grid-connected system is also analysed. The stability analysis of multi-inverters grid-connected system can provide theoretical and engineering guidance for construction of EVs power station system.

\section{REFERENCES}

[1] B Sun, T. Dragicevic, F. D. Freijedo, J. C. Vasquez, J. M. Guerrero, “A control algorithm for electric vehicle fast charging stations equipped with flywheel energy storage systems", IEEE Trans. Power Electronics, vol. 31, no. 4, pp. 6674-6685, 2016. DOI: 10.1109/TPEL.2015.2500962.

[2] Z. Xu, W. Su, Z. Hu, Y. Song, H. Zhang, "A hierarchical framework for coordinated charging of plug-in electric vehicles", IEEE Trans. on Smart Grid, vol. 7, no. 1, pp. 428-438, 2016. DOI: 10.1109/TSG.2014.2387436.

[3] S. Rivera, Bin Wu, "Electric vehicle charging station with an energy storage stage for split-DC bus voltage balancing", IEEE Trans. on Power Electronics, vol. 3, no. 3, pp. 2376-2386, 2017. DOI: 10.1109/TPEL.2016.2568039.

[4] M. R. Sarker, H. Pandzic, M. A. Ortega-Vazquez, "Optimal operation and services scheduling for an electric vehicle battery swapping station”, IEEE Trans. Power Systems, vol. 30, no. 2, pp. 901-910, 2015. DOI: 10.1109/TPWRS.2014.2331560.

[5] J. H. R. Enslin, P. J. M. Heskes, "Harmonic interaction between a large number of distributed power inverters and the distribution network", IEEE Trans. Power Electronics, vol. 19, no. 6, pp. 1586-1593, 2004. DOI: 10.1109/TPEL.2004.836615.

[6] Jinwei He, Yun Wei Li, D. Bosnjak, B. Harris, "Investigation and active damping of multiple resonances in a parallel-inverter based microgrid", IEEE Trans. Power Electronics, vol. 20, no. 1, pp. 234-246, 2013. DOI: 10.1109/TPEL.2012.2195032.

[7] Zhiyong Chen, An Luo, Yandong Chen, Mingshen Li, "Resonance features of multi-paralleled grid-connected inverters and its damping method", in Int. Power Electronics and Application Conf. and Exposition, 2014, pp. 120-125. DOI: 10.1109/PEAC.2014.7037840.

[8] A. Kotsopoulos, P. J. M. Heskes, M. J. Jansen, "Zero-crossing distortion in grid-connected PV inverters", IEEE Trans. Industrial Electronics, vol. 52, no. 2, pp. 558-565, 2005. DOI: 10.1109/TIE.2005.844254.

[9] J. L. Agorreta, M. Borrega, J. Lopez, L. Marroyo, "Modeling and control of N-paralleled grid-connected inverters with LCL filter coupled due to grid impedance in PV plants", IEEE Trans. on Power Electronics, vol. 26, no. 3, pp. 770-785, 2011. DOI: 10.1109/TPEL.2010.2095429.

[10] Byungcho Choi, Dongsoo Kim, Donggyu Lee, Seungwon Choi, Jian Sun, "Analysis of input filter interactionsin switching power converters", IEEE Trans. on Power Electronics, vol. 22, no. 2, pp. 452-460, 2007. DOI: 10.1109/TPEL.2006.889925.

[11] Xiaogang Feng, Jinjun Liu, Fred Lee, "Impedance specifications for stable DC distributed power systems", IEEE Trans. on Power Electronics, vol. 17, no. 2, pp. 157-162, 2002. DOI: 10.1109/63.988825.

[12] P. A. Dahono, "A control method to damp oscillation in the input LC filter", in 33rd Annual IEEE Power Electronics Specialists Conf., 2002, pp. 1630-1635. DOI: 10.1109/PSEC.2002.1023044. 